\title{
Notch Activation Induces Endothelial Cell Senescence and Pro- inflammatory Response: Implication of Notch Signaling in Atherosclerosis
}

\author{
Zhao-Jun Liu'1,2, Yurong Tan ${ }^{1}$, Gary W. Beecham ${ }^{3}$, David M. Seo ${ }^{3,4}$, Runxia Tian ${ }^{1}$, Yan Li ${ }^{1}$, \\ Roberto I. Vazquez-Padron ${ }^{1}$, Margaret Pericak-Vance ${ }^{3}$, Jeffery M. Vance ${ }^{3}$, Pascal J. \\ Goldschmidt-Clermont ${ }^{3}$, Alan S. Livingstone ${ }^{1}$, and Omaida C. Velazquez ${ }^{1,2,3,{ }^{*}}$ \\ ${ }^{1}$ Department of Surgery, University of Miami/Miller School of Medicine, University of Miami, \\ Miami, FL 33136 \\ ${ }^{2}$ Sylvester Comprehensive Cancer Center, University of Miami, Miami, FL 33136 \\ 3John P. Hussman Institute for Human Genomics, University of Miami, Miami, FL 33136 \\ ${ }^{4}$ Department of Medicine, University of Miami/Miller School of Medicine, University of Miami, \\ Miami, FL 33136
}

\begin{abstract}
Objective-Notch signaling plays pivotal roles in the pathogenesis of vascular disease. However, little is known about its role in atherosclerosis. We sought to investigate the potential involvement of the Notch signaling in atherosclerosis.
\end{abstract}

Methods-Expression of Notch pathway components in mouse and human aorta with or without atherosclerosis plaque was examined by immuno-histochemistry. Expression of Notch target genes in young versus aged human endothelial cells (EC) was examined by PCRArray and immunoblot. In vitro loss- and gain-of-function approaches were utilized to evaluate the role of Notch signaling in inducing EC senescence and secretion of pro-inflammatory cytokines by ProteinArray. Notch gene profile was studied in 1054 blood samples of patients with coronary artery disease (CAD). Genotyping was performed using the Genome-Wide Single Nucleotide Polymorphism (SNP) Array.

Results-Notch pathway components were upregulated in luminal EC at atherosclerotic lesions from mouse and human aortas. In addition, the Notch pathway was activated in aged but not young human EC. Enforced Notch activation resulted in EC senescence and significantly upregulated expression of several molecules implicated in the inflammatory response (IL-6/IL-8/ IL-1a/RANTES/ICAM-1). The upregulated IL-6 was partially responsible for mediating leukocyte transendothelial migration. Genetic association analysis detected, of 82 SNPs across 6 Notch pathway genes analyzed, 4 SNPs with nominal association with CAD burden.

(C) 2012 Elsevier Ireland Ltd. All rights reserved.

"Corresponding author: Omaida C. Velazquez, MD, FACS, Professor, Chief, Division of Vascular \& Endovascular Surgery, Department of Surgery, Leonard M. Miller school of Medicine, The University of Miami, 1611 NW 12th Avenue, East Tower 3016, Miami, FL 33101-6310, Phone: 305-585-5284, Fax: 305-585-8569, ovelazquez@med.miami.edu.

Conflict of Interest: None declared.

Publisher's Disclaimer: This is a PDF file of an unedited manuscript that has been accepted for publication. As a service to our customers we are providing this early version of the manuscript. The manuscript will undergo copyediting, typesetting, and review of the resulting proof before it is published in its final citable form. Please note that during the production process errors may be discovered which could affect the content, and all legal disclaimers that apply to the journal pertain. 
Conclusion-Notch pathway is activated in luminal EC at atherosclerotic plaques and results in pro-inflammatory response and senescence of EC. Notch signaling may be linked to human CAD risk. These findings implicate a potential involvement of Notch signaling in atherosclerosis.

\section{Keywords}

Notch; Endothelial cells (EC); Atherosclerosis; EC Senescence; Vascular inflammation

\section{Introduction}

Inflammation has emerged as a major driving force of atherosclerosis development and progression [1] [2]. In the early stage of atherosclerosis, the injured endothelium becomes activated during the inflammatory process involving adhesion/accumulation of platelets and recruitment/infiltration of the inflammatory subset of monocytes/macrophages to the arterial wall. Infiltrated monocytes/macrophages eventually transform into foam cells, leading to the development of characteristic atherosclerosis plaque lesions [3]. Inflammatory cells within the plaque secrete matrix proteases that degrade extracellular matrix proteins and weaken the protective fibrous cap of the atheroma, leading to thrombosis and the occurrence of acute coronary syndromes [4]. Some circulating cells, such as endothelial progenitor cells (EPC) are thought to potentially counter the destructive effects by re-endothelializing the denuded vascular surface [5]. In addition, some "by-products" created in the process of inflammation and vascular injury, i.e. platelet-derived microparticles (PMPs) and apoptotic bodies from senescent EC, contribute to boost the potential of EPC for restoring endothelial integrity [6] [7]. When the pro-atherogenesis risk factors overwhelm the ability of EPC and other repair mechanisms to maintain arterial homeostasis, the inflammation cascades are self-amplified and compromise the stability of the arterial wall. Atherosclerosis could thus be envisioned as an imbalance between vascular repair and vascular damage inflammation-related cascades.

Aging is one of the most well established risk factors for atherosclerosis. At the cellular level, aging of vascular EC leads to senescence. Senescence is characterized by specific changes in cell morphology and gene expression, which reduce EC function [8] and thus are proposed to be pro-atherogenic [9] [10]. Although mitotically inactive, senescent cells are not physiologically inert: they secrete pro-inflammatory cytokines, growth factors and degradative enzymes [11], and this could promote or contribute to the pathogenesis of atherosclerosis [12]. In fact, EC senescence is often accompanied by a low-grade chronic up-regulation of certain pro-inflammatory responses [13].

Notch signaling plays an essential role in vascular development [14] [15] [16]. It is also critical for post-natal vascular diseases. For instance, mutations of the Notch3 gene cause a hereditary vascular degenerative disease[17] while activation of Notch1 in smooth muscle cells mediates neointimal formation after vascular injury [18]. Delta-like 4 (DLL4)-induced Notch activation has been observed in macrophages within atherosclerotic plaques [19]. Consistently, systemic administration of $\gamma$-secretase inhibitor (GSI), which blocks the Notch activation, reduces the progression of atherosclerotic plaques in $\mathrm{ApoE}^{-/-}$mice by altering the immune response [20]. However, Notch's role in human atherosclerosis remains largely unexplored.

In this study, we provide evidence that links the Notch signaling with EC senescence and inflammation and implies a potential involvement of Notch signaling in conferring risk for human atherosclerosis. 


\section{Materials and Methods}

\section{Cells and tissues}

Human microvascular endothelial cells (HMVEC), provided by Dr. D. Fraker, University of Pennsylvania, were isolated from human dermis. Human aorta endothelial cells (HAEC) were purchased from Lonza (CC-2535, Allendale, NJ). HMVEC and HAEC were cultured as described [21] [22]. Human monocyte cells, THP-1 were purchased from ATCC (Manassas, VA), and cultured as recommended. 293T and NIH/3T3 cells were cultured in DMEM (Invitrogen) supplemented with 10\% fetal bovine serum (FBS). Human aorta and blood samples were collected from patients/donors following the guideline of using human tissues approved by the committee of institutional review board (IRB) of the University of Miami. Biopsies from different portions of aortas with and without atherosclerosis plaque were obtained from human organ donors and operative cases in human subjects consented to donate tissue for research under protocol IRB \# 20080425. Murine aortas were harvested from $\mathrm{ApoE}^{-/-}$mice fed with or without high-fat diet (TD.88137 Adjusted Calories Diet (42\% from fat) from Harlan, Tampa, FL) for 16 weeks and C57 BL6 mice (Charles River, Wilmington, $\mathrm{MA}$ ) which were euthanized in $\mathrm{CO}_{2}$ gas chamber. Animal experiments were approved by the Institute Animal Care and Use Committee (IACUC\# 09-074) of the University of Miami.

\section{Recombinant lentiviruses}

Methods for generation of GFP/lenti (control) and $\mathrm{N}^{\mathrm{IC}}$-GFP/lenti (Notch1 intracellular domain) were described previously [23]. Production of pseudotyped lentivirus was achieved by co-transfecting $293 \mathrm{~T}$ cells with three plasmids as described [24]. The lentiviruses collected 48 hours post-transfection displayed titers of around $10^{7}$ transducing units $/ \mathrm{ml}$ in $\mathrm{NIH} / 3 \mathrm{~T} 3$ cells. To infect target cells by lentiviruses, cells were exposed six hours to virus with MOI (multiplicity of infection) 5 in the presence of $4 \mu \mathrm{g} / \mathrm{ml}$ polybrene (Sigma-Aldrich, St. Louis, MO). Cells were analyzed for protein expression by Immunoblot or pooled for subsequent analysis as indicated in individual experiments.

\section{ELISA}

Concentration of IL-6 and IL-83 in the cell lysates and supernatant of cell cultures was measured by ELISA as detailed in Supplemental Materials.

\section{Immunoblotting and immunohistochemistry (IHC)}

Immunoblotting and IHC were performed as previously described [21] [25]. See Supplemental Materials for details.

\section{Cell growth, senescence and telomerase assay}

Cell growth of HAEC was determined by cell counting. $5 \times 10^{4}$ cells were plated in 6-well plate and cultured in complete EGM2 medium. Cell number was counted every day for 4 days. EC senescence was detected using Senescence Cells Histochemical Staining Kit (Sigma CS0030) and telomerase activity in cells were measured by TRAPeze ${ }^{\circledR} X L$ Telomerase Detection Kit (S7707, Millipore, Billerica, MA), respectively, based on the manufacturers' protocols.

\section{PCR-Array}

The Human Notch Signaling Pathway RT ${ }^{2}$ Profiler ${ }^{\mathrm{TM}}$ PCR Array quantitatively profiles the expression of 84 genes involved in Notch signaling (\# PAHS-059, SABiosciences). Total RNA was extracted from cells using Trizol ${ }^{\circledR}$ (Invitrogen) and cDNA was synthesized using $\mathrm{RT}^{2}$ First Strand Kits (SABiosciences). PCR array was carried out according to the 
manufacturer's protocol. The threshold cycle $(\mathrm{Ct})$ values were used to plot a standard curve. All samples were normalized to the relative levels of $\beta$-actin, and results are expressed as fluorescence intensity in relative levels.

\section{Protein Array}

Protein Array analysis was employed to examine the expression of a panel of soluble factors produced by HMVEC-N ${ }^{I C}$-GFP versus HMVEC-GFP cells. The culture supernatants from equal numbers of HMVEC-N ${ }^{\mathrm{IC}}-$ GFP and HMVEC-GFP cells, which were cultured in basic EGM-2 medium (serum-free and growth factor-free) for 12 hours, were collected and subjected to TranSignal ${ }^{\mathrm{TM}}$ Human Cytokine Antibody 3.0 (Panomics, Fremont, CA) analysis, which allows detection of 36 different proteins in total. The samples tested were further normalized based on the total protein amount from cell lysates. Experiments were repeated three times.

\section{Transendothelial migration assay}

The assay was conducted in transwell and see Supplemental Materials for details.

\section{Statistical analysis}

All data is expressed as mean \pm SD. Statistical analysis was carried out using two-tails Student's $t$-test. $P$-value $₫ 0.05$ was considered to be statistically significant.

\section{Results}

\section{The Notch pathway is activated in luminal EC at atherosclerotic sites of mouse and human aortas}

To explore the potential role of Notch signaling in atherosclerosis, we conducted IHC analysis to examine the expression patterns of several Notch receptors, ligands and target genes in atherosclerotic versus non-atherosclerotic sites from aortas of $\mathrm{ApoE}^{-/-}$mice fed with high fat diet versus non-atherosclerotic aortas of $\mathrm{ApoE}^{-/-}$mice fed with low fat diet and C57 BL6 mice (n=3/group). Expression of Notch1 (Figure 1A, 1C), Notch3, Jagged1 and DLL4 (Supplemental Figure 1-3) was significantly higher in atherosclerotic lesions of aortas in $\mathrm{ApoE}^{-/-}$mice fed with high-fat diet compared to non-atherosclerotic sites of aortas in $\mathrm{ApoE}^{-/-}$mice fed with low-fat diet. Consistently, levels of Hes1 were significantly higher in atherosclerotic lesions compared to non-atherosclerotic aortas from control mice (Figure $1 \mathrm{C}$ and Supplemental Figure 4). Up-regulated expression of Hey1, an indication of Notch pathway activation, was observed in luminal EC in aortic lesion of $\mathrm{ApoE}^{-/-}$mice fed with high fat diet. The endothelial phenotype of these luminal cells was confirmed by IHC showing positive staining with anti-VEGFR2 antibody (Figure 1B). IHC data for other Notch pathway components were not shown due to the poor quality of antibodies for IHC. These data suggested that the Notch pathway is activated in atherosclerotic lesions.

We also evaluated the expression patterns of several Notch pathway components in human aortic biopsies obtained from 7 donors. The expression of Notch 4 was increased in atherosclerotic lesions compared to non-atherosclerotic sites in all 7 human aortic biopsy samples (Figure1C and Supplemental Figure 5). Notch 1 was also up-regulated in atherosclerotic lesions compared to non-atherosclerotic sites (Supplemental Figure 6), although Notch 4 levels changed most dramatically. Similarly, the expression of Notch ligands, including Jagged2 and DLL3 (Supplemental Figure 7-8), and levels of Notch target genes were also increased at atherosclerosis plaques. Hey1 (Supplemental Figure 9) and Hes1 (Figure 1C and Supplemental Figure 10) expression were significantly higher in atherosclerotic lesions compared to non-atherosclerotic sites. Heslexpression was detected in luminal EC, vascular smooth cells and inflammatory cells at atherosclerotic sites. IHC 
data for other Notch pathway components were not shown due to the poor quality of antibodies for IHC. Overall, results from the human and mouse aortic samples (Figure 1C) suggest that the Notch pathway is activated in luminal EC of atherosclerotic lesions and imply a potential involvement of the Notch signaling in atherosclerosis.

To further investigate the potential involvement of Notch in atherosclerosis, we conducted a genetic association study by analysis of our existing SNP genotyping database established by testing 1054 blood samples of patients with coronary artery disease (CAD). Genetic association analysis detected, of 82 SNPs across 6 Notch pathway genes analyzed, 4 SNPs with nominal association with CAD burden (Supplemental Table-1 and -2). It suggests that Notch signaling may be linked to human CAD risk.

\section{Activation of Notch1 signaling inhibits EC growth and induces EC senescence}

To investigate the role of Notch signaling in EC biology, we tested the effect of enforced activation of Notch1 signaling on HAEC proliferation (expression of $\mathrm{N}^{\mathrm{IC}}$ in $\mathrm{N}^{\mathrm{IC}}$-GFP/ HAEC versus GFP/HAEC was shown in Insert of Figure 2A) and observed that Notch activation resulted in significantly retarded cell growth and induced an enlarged, flattened and dendritic senescent morphology (Figure 2A). We therefore studied whether Notch pathway activation induces EC senescence. Human EC, including HMVEC and HAEC, expressing the active form of Notch1 along with GFP ( ${ }^{\mathrm{IC}}$-GFP/HMVEC and $\mathrm{N}^{\mathrm{IC}}-\mathrm{GFP} /$ HAEC) versus control cells (GFP/HMVEC and GFP/HAEC) were subjected to cell senescence assay (a similar expression pattern of $\mathrm{N}^{\mathrm{IC}}$ in $\mathrm{N}^{\mathrm{IC}}$-GFP/HMVEC versus GFP/ HMVEC as that of $\mathrm{N}^{\mathrm{IC}}$-GFP/HAEC versus GFP/HAEC was observed (data not shown). Notch activation resulted in EC senescence since more senescent $\left(\beta\right.$-gal $\left.{ }^{+}\right)$cells were detectable from $\mathrm{N}^{\mathrm{IC}}$-GFP/HMVEC and $\mathrm{N}^{\mathrm{IC}}$-GFP/HAEC than GFP/HMVEC and GFP/ HAEC (Figure 2B). Notably, Notch activation induced more cell senescence in HAEC than in HMVEC. To further investigate whether Notch-induced EC senescence is a telomere shortening-induced replicative senescence, we examined telomerase activity in HMVEC. It was found that $\mathrm{N}^{\mathrm{IC}}$-GFP/HMVEC displayed significantly lower telomerase activity compared to GFP/HMVEC (Figure 2C). Thus, these data demonstrate that activation of Notch1 signaling causes EC dysfunction by inducing cell senescence.

\section{Expression of HeyL, a Notch target gene, is elevated in aged versus young EC}

The findings of the involvement of the Notch signaling in EC senescence led us to investigate the potential correlation between the status of Notch signaling and EC aging. Expression of 84 Notch pathway-related genes was examined quantitatively using $R T^{2}$ PCR-Array. HMVEC which are primary human cells usually become senescence around passage \# 15 under our culture condition. The young (passage \# 5) and old (passage \# 12) HMVEC were compared. Total RNA was extracted and subjected to be tested using $R T^{2}$ PCR-Array. It was found that expression of HeyL gene was significantly higher in aged versus young EC (Figure 3A). Due to the difficulty in getting high quality of RNA from aged cells, many Notch pathway component genes were undetectable in high-passage aged cells. Increased expression of HeyL protein in aged HMVEC was also confirmed by immunoblot analysis (Figure 3B). These data indicate that the HeyL expression is correlated with EC aging, and suggest a potential involvement of Notch pathway activation in this dynamic process of aging-related cell senescence.

\section{Activation of Notch1 signaling upregulates expression of pro-inflammatory cytokines/ chemokines and ICAM-1 in human EC}

To test whether Notch activation participates in regulating cytokine production in EC, we conducted a ProteinArray ${ }^{\circledR}$ analysis to examine the expression of a panel of cytokines by $\mathrm{N}^{\mathrm{IC}}$-GFP/HMVEC versus GFP/HMVEC. The culture supernatants from starved $\mathrm{N}^{\mathrm{IC}}-\mathrm{GFP} /$ 
HMVEC and GFP/HMVEC, which were cultured in basal EGM2 medium (serum-free and growth factor-free) for 12 hours, were collected and subjected to TranSignal ${ }^{\mathrm{TM}}$ Human Cytokine Antibody Array 3.0 analyses, which allow detection of 36 different proteins. Of 36 proteins, levels of IL-6, IL-8, IL-1a, RANTES and cell adhesion molecule ICAM-1 were up-regulated in microvascular EC ( $\mathrm{N}^{\mathrm{IC}}-\mathrm{GFP} / \mathrm{HMVEC}$ compared to GFP/HMVEC, Figure $4 \mathrm{~A}$ and Supplemental Table-3). Notably, these upregulated proteins are all inflammationrelated, suggesting that activation of Notch1 pathway results in the production of proinflammatory factors in EC. Increased expression of IL-6, IL-8 in cell lysates of $\mathrm{N}^{\mathrm{IC}}-\mathrm{GFP} /$ HMVEC was confirmed by Quantikine Human IL-6 and IL-8 ELISA (RANTES and IL-1a were undetectable by ELISA in HMVEC) (Figure 4B), and upregulated ICAM-1 was validated by immunoblot (Figure 4C). Blockade of Notch signaling by dominant negativeMastermind-like-1 (DN-MAML-1) inhibited production of IL-6 and IL-8 (Figure 4B). Notably, DN-MAML1 was able to reverse Notch signaling-up-regulated IL-6 and IL-8 to its basal level (in the case of IL-6) or close to its basal level (in the case of IL-8), but unable to bring about it below the basal levels, suggesting either that the basal level of IL-6 and IL-8 expression is not solely regulated by the Notch signaling in EC (if the DN-MAML1 could completely block the Notch signaling), or that DN-MAML1 cannot completely block Notch signaling. Significant upregulation of ICAM-1 in the cell lysates (Figure 4C) and IL-6, IL-8 and RANTES in the supernatants of cell cultures (Figure 4D) were also observed in aortic $\mathrm{EC}\left(\mathrm{N}^{\mathrm{IC}}-\mathrm{GFP} / \mathrm{HAEC}\right.$ compared to GFP/HAEC). Increased levels of these pro-inflammatory cytokines in HAEC were even higher than that in HMVEC. These data demonstrate that Notch pathway activation up-regulates a panel of pro-inflammatory factors and adhesion molecules in human EC, pointing to a potential involvement of the Notch signaling family in vascular diseases characterized by inflammation such as atherosclerosis.

\section{Notch1 induces increased monocyte transendothelial migration, mediated in part by IL-6}

To test the hypothesized role of IL-6 in mediating monocyte transendothelial migration, we examined the direct effect of $\gamma \mathrm{hIL}-6$ on THP-1 (a human monocytic leukaemia cell line with capability to differentiate into macrophage-like cells) transendothelial migration in a transwell system. HMVEC monolayers were cultured in the upper chamber of transwell inserts. Application of $\gamma \mathrm{hIL}-6$ to the lower chamber efficiently facilitated monocyte transendothelial migration compared to BSA control, confirming that IL-6 is a chemotaxin for monocytes. Secondly, $\mathrm{N}^{\mathrm{IC}}-\mathrm{GFP} / \mathrm{HMVEC}$ and GFP/HMVEC were plated to form monolayers on the lower chamber to test leukocyte transendothelial migration. Culture supernatants from a separately cultured $\mathrm{N}^{\mathrm{IC}}$-GFP/HMVEC and GFP/HMVEC were added to the lower chamber of the transwells. Compared to GFP/HMVEC, $\mathrm{N}^{\mathrm{IC}}-\mathrm{GFP} / \mathrm{HMVEC}$ monolayers resulted in significantly more monocytes transmigration from the upper chamber, indicating that $\mathrm{N}^{\mathrm{IC}}-\mathrm{GFP} / \mathrm{HMVEC}$ are more "chemotactic" to monocytes. IL-6 appears to be partially responsible for this "chemotactic" effect of $\mathrm{N}^{\mathrm{IC}}-\mathrm{GFP} / \mathrm{HMVEC}$, since blockade of IL- 6 by adding neutralizing antibody $(2 \mu \mathrm{g} / \mathrm{ml})$ to the transwell was able to partially inhibit, in a statistically significant extent, the Notch-induced monocyte transendothelial migration (Figure 5). The inhibitory effect was antibody dosage-dependent, suggesting that the inhibition is IL-6 specific. Overall, these results demonstrate that Notch signaling-induced cytokines/chemokines, such as IL-6, in EC are responsible for mediating monocyte transendothelial migration in vitro, thus increasing the evidence that Notch activation induces an EC pro-inflammatory response.

\section{Discussion}

Our findings demonstrate that constitutive activation of Notch1 signaling induces EC senescence. It is consistent with a previous report by Venkatesh et al [26]. Consistent with those results, HeyL, a Notch down-stream target, is elevated in aged compared to young EC. 
Notch activation also triggers EC inflammatory response by up-regulating expression of a panel of pro-inflammatory cytokines/chemokines and adhesion molecules in EC. These findings unveil a novel function of Notch1 signaling in EC biology and may shed light on the mechanism whereby Notch signaling may contribute to some age-related vascular diseases characterized by chronic inflammation, such as human atherosclerosis. A strong relevance of these in vitro biologic findings to the human atherosclerosis disease is highly suggested by the data that some Notch receptors, ligands, and target genes are upregulated in luminal EC at mouse and human aortic atherosclerosis plaque and by the genetic association analysis which detected 4 SNPs in 6 tested Notch pathway genes that may confer CAD risk.

Notch signaling affects several biologic functions in vascular EC, including cell proliferation, differentiation and apoptosis. Our previous studies revealed a role of Notch signaling in inducing "differentiation-associated growth arrest" in HIAEC [21] under certain specific experimental settings, for instance, using an in vitro angiogenesis model wherein EC favor formation and stabilization of vascular networks in a given period of time. Our new findings are concordant with previous observation and significantly expand our understanding on the role of the Notch signaling in regulating EC biology and the functional connection between Notch signaling and EC senescence. These findings suggest an intriguing new concept that Notch signaling may be important in the pathogenesis of common age-related vascular diseases such as atherosclerosis.

We observed that multiple Notch receptors, including Notch1, Notch3 and Notch4, are upregulated in atherosclerotic lesions. It is unclear whether individual Notch members exert distinct or identical functions. We focus on Notch1 signaling in the in vitro experiments and our data provide proof-of-concept that Notch signaling carries a specific functional effect on EC biology and potentially, on atherogenesis.

The observation that increased expression of Notch pathway components is a feature of atherosclerotic plaques supports the notion that Notch-induced vascular inflammation is more likely to be involved in the pathogenesis of atherogenesis. It does not, however, exclude the possibility that Notch may participate in the recruitment of EPC for arterial repair if it occurs during the early phase of vascular injury. CXCL12/SDF-1a and its cognate receptor CXCR4 are known to be involved in EPC recruitment [27] [28] [29]. It may be worthwhile to test whether Notch activation induces EC to express CXCL12/ SDF-1a and/or CXCR4, and more importantly, to characterize the biological significance of Notch-induced vascular inflammation in arterial repair versus atherogenesis.

Activation of DLL4-Notch pathway in macrophages within atherosclerotic plaques has been previously reported [19]. We also observed that expression of several Notch pathway components were increased in luminal EC as well as vascular smooth cells (VSMC) and inflammatory cells at atherosclerotic sites. Likely, there are crosstalk among EC, VSMC and inflammatory cells. However, the specific functional significance of Notch activation in different types of cells within atherosclerotic lesion is unknown. It is possible that all contribute, through various mechanisms and their crosstalk, to atherogenesis. The effect of GSI on reduction of atherosclerosis in $\mathrm{ApoE}^{-/-}$mice [20], may rely on suppression of panNotch pathway and target multiple types of cells, including EC, smooth muscle cells and inflammation cells, in the injured arteries.

In summary, our data demonstrate that Notch signaling is a control element of cell senescence and inflammatory response in human EC. In consideration of the additional findings that SNPs in six tested Notch pathway genes may confer CAD risk, that activation of Notch signaling is observed in luminal EC at aortic lesions of atherosclerosis, and that age-related chronic inflammation is a major risk factor in this type of vascular disorder, our 
study strongly implicates a functional involvement of the Notch signaling in atherosclerosis. Further work is warranted to explore if a direct causal linkage exists between the Notch signaling and development and progression of atherosclerosis.

\section{Supplementary Material}

Refer to Web version on PubMed Central for supplementary material.

\section{Acknowledgments}

We thank Dr. D. Fraker for HMVECs and Dr. G. McNamara for quantification of IHC data. This work was supported by grants from the National Institutes of Health (R01DK-071084 and R01GM081570, to OCV).

\section{References}

1. Ross R. Atherosclerosis--an inflammatory disease. N Engl J Med. 1999; 340:115-126. [PubMed: 9887164]

2. Libby P. Inflammation in atherosclerosis. Nature. 2002; 420:868-874. [PubMed: 12490960]

3. Paoletti R, Gotto AM Jr, Hajjar DP. Inflammation in atherosclerosis and implications for therapy. Circulation. 2004; 109:III20-26. [PubMed: 15198962]

4. Libby P, Ridker PM, Maseri A. Inflammation and atherosclerosis. Circulation. 2002; 105:11351143. [PubMed: 11877368]

5. Goldschmidt-Clermont PJ, Creager MA, Losordo DW, Lam GK, Wassef M, Dzau VJ. Atherosclerosis 2005: recent discoveries and novel hypotheses. Circulation. 2005; 112:3348-3353. [PubMed: 16301361]

6. Mause SF, Ritzel E, Liehn EA, Hristov M, Bidzhekov K, Muller-Newen G, Soehnlein O, Weber C. Platelet microparticles enhance the vasoregenerative potential of angiogenic early outgrowth cells after vascular injury. Circulation. 2010; 22:495-506. [PubMed: 20644015]

7. Hristov M, Zernecke A, Liehn EA, Weber C. Regulation of endothelial progenitor cell homing after arterial injury. Thromb Haemost. 2007; 98:274-277. [PubMed: 17721606]

8. Wagner M, Hampel B, Bernhard D, Hala M, Zwerschke W, Jansen-Durr P. Replicative senescence of human endothelial cells in vitro involves G1 arrest, polyploidization and senescence-associated apoptosis. Exp Gerontol. 2001; 36:1327-1347. [PubMed: 11602208]

9. Serrano AL, Andres V. Telomeres and cardiovascular disease: does size matter? Circ Res. 2004; 94:575-584. [PubMed: 15031270]

10. Edo MD, Andres V. Aging, telomeres, and atherosclerosis. Cardiovasc Res. 2005; 66:213-221. [PubMed: 15820190]

11. Chen J, Goligorsky MS. Premature senescence of endothelial cells: Methusaleh's dilemma. Am J Physiol Heart Circ Physiol. 2006; 290:H1729-1739. [PubMed: 16603702]

12. Minamino T, Komuro I. Vascular cell senescence: contribution to atherosclerosis. Circ Res. 2007; 100:15-26. [PubMed: 17204661]

13. Minamino T, Miyauchi H, Yoshida T, Ishida Y, Yoshida H, Komuro I. Endothelial cell senescence in human atherosclerosis: role of telomere in endothelial dysfunction. Circulation. 2002; 105:1541-1544. [PubMed: 11927518]

14. Herbert SP, Huisken J, Kim TN, Feldman ME, Houseman BT, Wang RA, Shokat KM, Stainier DY. Arterial-venous segregation by selective cell sprouting: an alternative mode of blood vessel formation. Science. 2009; 326:294-298. [PubMed: 19815777]

15. Gianni-Barrera R, Trani M, Reginato S, Banfi A. To sprout or to split? VEGF, Notch and vascular morphogenesis. Biochem Soc Trans. 2011; 39:1644-1648. [PubMed: 22103501]

16. Alva JA, Iruela-Arispe ML. Notch signaling in vascular morphogenesis. Curr Opin Hematol. 2004; 11:278-283. [PubMed: 15314528]

17. Joutel A, Tournier-Lasserve E. Notch signalling pathway and human diseases. Semin Cell Dev Biol. 1998; 9:619-625. [PubMed: 10075489] 
18. Li Y, Takeshita K, Liu PY, Satoh M, Oyama N, Mukai Y, Chin MT, Krebs L, Kotlikoff MI, Radtke F, Gridley T, Liao JK. Smooth muscle Notch1 mediates neointimal formation after vascular injury. Circulation. 2009; 119:2686-2692. [PubMed: 19433762]

19. Fung E, Tang SM, Canner JP, Morishige K, Arboleda-Velasquez JF, Cardoso AA, Carlesso N, Aster JC, Aikawa M. Delta-like 4 induces notch signaling in macrophages: implications for inflammation. Circulation. 2007; 115:2948-2956. [PubMed: 17533181]

20. Aoyama T, Takeshita K, Kikuchi R, Yamamoto K, Cheng XW, Liao JK, Murohara T. gammaSecretase inhibitor reduces diet-induced atherosclerosis in apolipoprotein E-deficient mice. Biochem Biophys Res Commun. 2009; 383:216-221. [PubMed: 19345673]

21. Liu ZJ, Shirakawa T, Li Y, Soma A, Oka M, Dotto GP, Fairman RM, Velazquez OC, Herlyn M. Regulation of Notch1 and D114 by vascular endothelial growth factor in arterial endothelial cells: implications for modulating arteriogenesis and angiogenesis. Mol Cell Biol. 2003; 23:14-25. [PubMed: 12482957]

22. Liu ZJ, Snyder R, Soma A, Shirakawa T, Ziober BL, Fairman RM, Herlyn M, Velazquez OC. VEGF-A and alphaVbeta3 integrin synergistically rescue angiogenesis via N-Ras and PI3-K signaling in human microvascular endothelial cells. FASEB J. 2003; 17:1931-1933. [PubMed: 14519669]

23. Hogan, B.; Beddington, R.; Costantini, F.; Lacey, E. Manipulating the Mouse Embryo: A Laboratory Manual. Cold Spring Harbor Laboratory Press; Plainview, N.Y: 1994.

24. Kobinger GP, Weiner DJ, Yu QC, Wilson JM. Filovirus-pseudotyped lentiviral vector can efficiently and stably transduce airway epithelia in vivo. Nat Biotechnol. 2001; 19:225-230. [PubMed: 11231554]

25. Balint K, Xiao M, Pinnix CC, Soma A, Veres I, Juhasz I, Brown EJ, Capobianco AJ, Herlyn M, Liu ZJ. Activation of Notch1 signaling is required for beta-catenin-mediated human primary melanoma progression. J Clin Invest. 2005; 115:3166-3176. [PubMed: 16239965]

26. Venkatesh D, Fredette N, Rostama B, Tang Y, Vary CP, Liaw L, Urs S. RhoA-mediated signaling in Notch-induced senescence-like growth arrest and endothelial barrier dysfunction. Arterioscler Thromb Vasc Biol. 2011; 31:876-882. [PubMed: 21273559]

27. Gallagher KA, Liu ZJ, Xiao M, Chen H, Goldstein LJ, Buerk DG, Nedeau A, Thom SR, Velazquez OC. Diabetic impairments in NO-mediated endothelial progenitor cell mobilization and homing are reversed by hyperoxia and SDF-1 alpha. J Clin Invest. 2007; 117:1249-1259. [PubMed: 17476357]

28. Liu ZJ, Velazquez OC. Hyperoxia, endothelial progenitor cell mobilization, and diabetic wound healing. Antioxid Redox Signal. 2008; 10:1869-1882. [PubMed: 18627349]

29. Liu ZJ, Tian R, An W, Zhuge Y, Li Y, Shao H, Habib B, Livingstone AS, Velazquez OC. Identification of E-selectin as a novel target for the regulation of postnatal neovascularization: implications for diabetic wound healing. Ann Surg. 2010; 252:625-634. [PubMed: 20881769] 


\section{Highlights}

- Notch pathway is activated in luminal EC at atherosclerotic plaques

- Notch pathway activation results in pro-inflammatory response and senescence of luminal EC

- Notch signaling may be linked to human CAD risk

- Involvement of Notch signaling in atherosclerosis 
Figure $1 \mathrm{~A}$

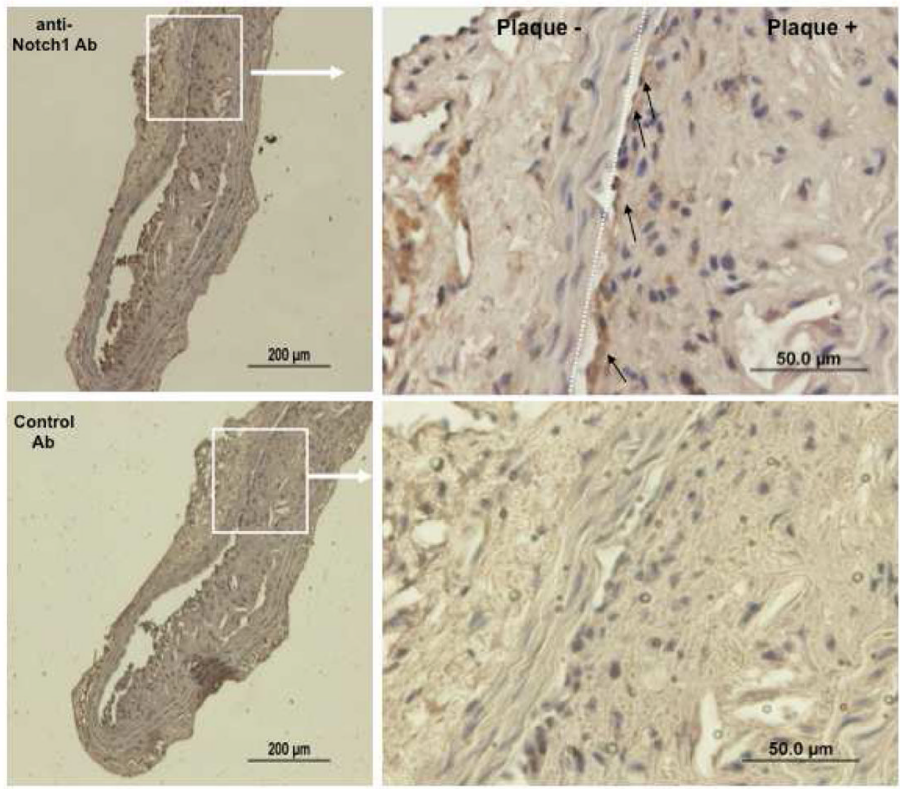

Figure 1B
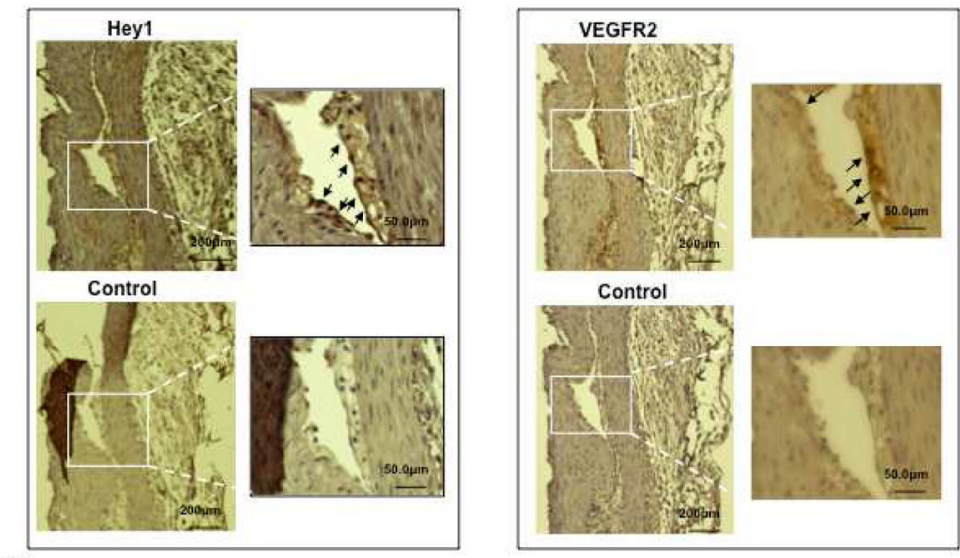

$1 C$

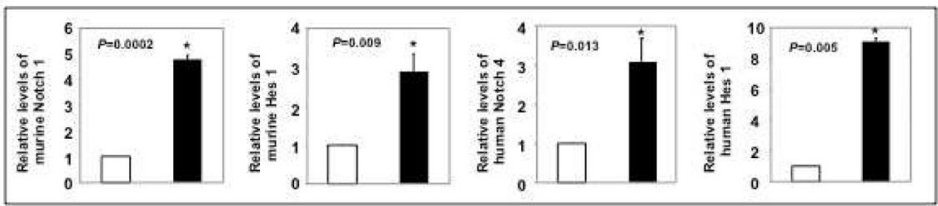

Figure 1.

Representative immunohistochemistry showing the upregulation of the Notch pathway components in atherosclerotic sites of aortas. (A) Expression patterns of Notch 1 in atherosclerotic versus non-atherosclerotic sites from aortas of $\mathrm{ApoE}^{-/}$mice fed with high fat diet respectively. (B) Endoluminal localization of the expression of Hey1 and VEGFR2 indicating a luminal EC intima layer that expresses Heyl in aortic lesion of $\mathrm{ApoE}^{-/-}$mice fed with high fat diet. (C) Relative expression of Notch 1 and Hes 1 in aortas of ApoE ${ }^{-/-}$ mice at endothelium of atherosclerotic versus non-atherosclerotic sites, and Notch 4, Hes 1 in patient aortas. Pixel intensity of brown IHC stained endothelium was calculated and normalized by setting the value from non-atherosclerotic sites as " 1 ". Data are presented as 
mean \pm SD of three independently stained IHC samples from individual donors or mice ( $n=3$ /group). White column: plaque + ; black column: plaque - . 

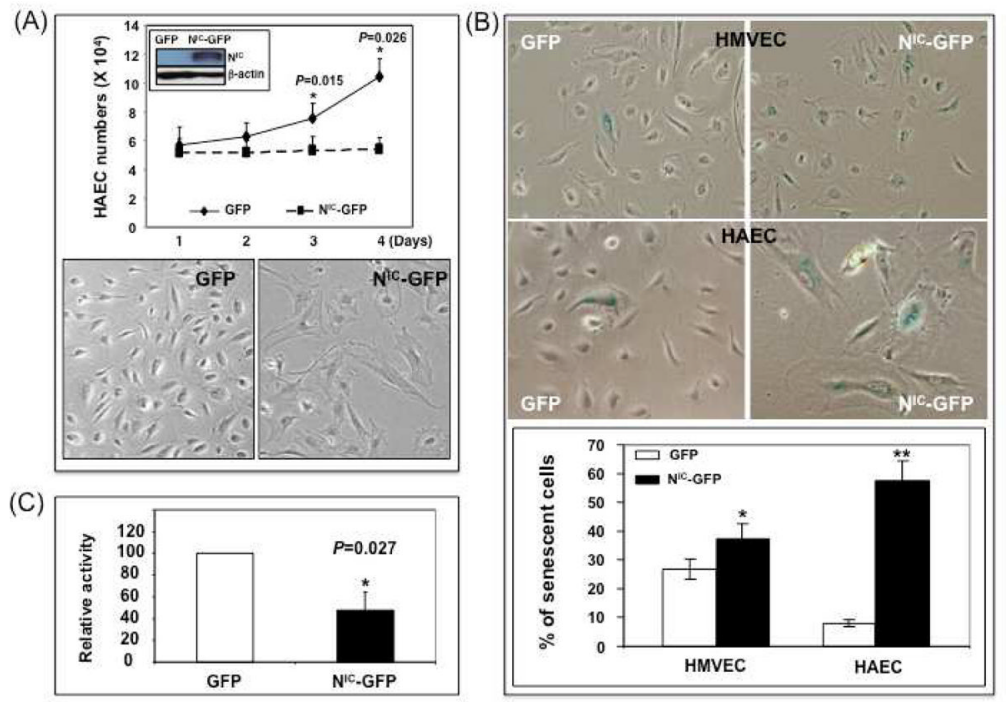

Figure 2.

Activation of Notch1 signaling Inhibits EC growth and induces EC senescence. (A) Constitutive activation of Notch1 suppressed HAEC proliferation (top), and induced a senescent morphology (bottom). Insert: expression of $\mathrm{N}^{\mathrm{IC}}$ in $\mathrm{N}^{\mathrm{IC}}-\mathrm{GFP} / \mathrm{HAEC}$ versus GFP/ HAEC. $\beta$-actin was used as loading control. (B) An increased number of senescent $\left(\beta\right.$-gal $\left.{ }^{+}\right)$ cells were detectable from $\mathrm{N}^{\mathrm{IC}}$-GFP/HMVEC and $\mathrm{N}^{\mathrm{IC}}$-GFP/HAEC versus GFP/HMVEC and GFP/HAEC, respectively. Top: representative images. Bottom: percentage of senescent $\left(\beta-\mathrm{gal}^{+}\right)$cells was shown. $* p<0.05$, $* * p<0.0001$. (C) Telomerase activity in $\mathrm{N}^{\mathrm{IC}}-\mathrm{GFP} /$ HMVEC versus GFP/HMVEC. Telomerase activity in control cells GFP/HMVEC was set as " $100 \%$ " for standardization. All data are presented as mean \pm SD of three independently performed experiments. 

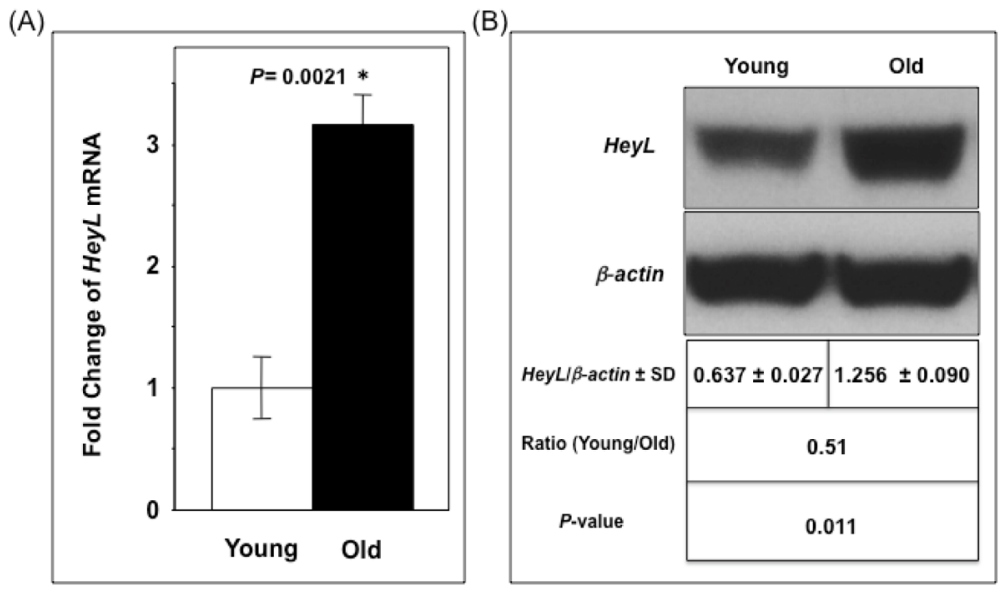

Figure 3.

Elevated expression of Notch target gene, HeyL, in aged EC. (A) Expression of HeyL mRNA was significantly higher in aged versus young HMVEC as measured by quantitative PCRArray. Experiments were repeated for three times and data are presented as mean \pm SD. (B) Increased expression of HeyL protein in aged HMVEC was confirmed by immunoblot analysis. $\beta$-actin was used as loading control. One representative result is shown. Quantitative data was based on three experiments. Both PCRArray and immunoblot experiments were repeated three times and reproducible, consistent patterns were observed. 
(A)

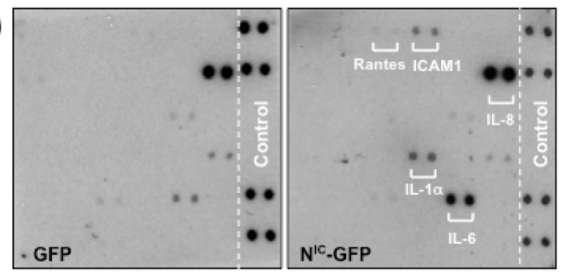

(B)

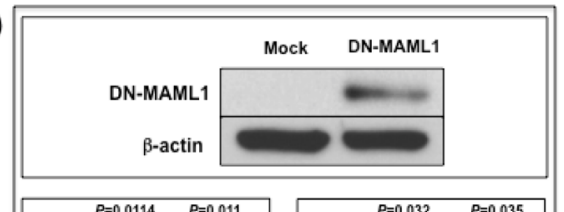

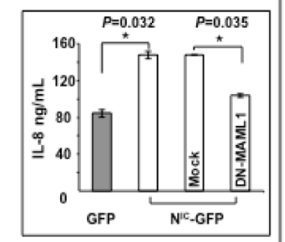

(C)

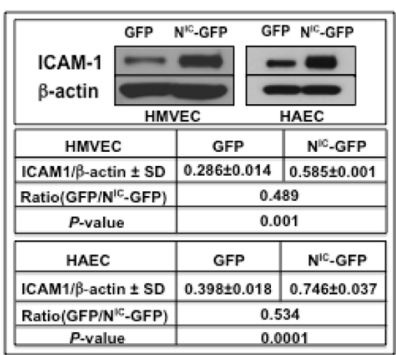

(D)

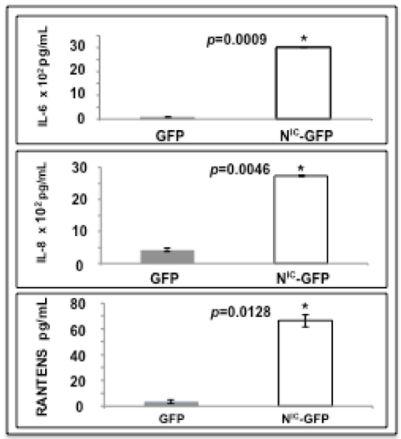

Figure 4.

Notch pathway activation induces expression of pro-inflammatory factors and ICAM-1 in human EC. (A) ProteinArray analysis of 36 proteins induced by $\mathrm{N}^{\mathrm{IC}}$-GFP/HMVEC versus GFP/HMVEC. Notch activation upregulated expression of IL-6, IL-8, IL-1a, RANTES and cell adhesion molecule ICAM-1. (B) Detection of IL-6 and IL-8 by ELISA. Cell lysates from $\mathrm{N}^{\mathrm{IC}}$-GFP/HMVEC versus GFP/HMVEC were normalized based on protein quantization and tested by Quantikine ${ }^{\circledR}$ IL-6 or IL-8 assay kit. Data are presented as mean \pm SD of three independently performed experiments. Blockade of Notch signaling by DNMAML-1 reversed expression patterns of IL-6 and IL-8 in $\mathrm{N}^{\mathrm{IC}}$-GFP/HMVEC. Cell lysates from DN-MAML-1-N ${ }^{\mathrm{IC}}$-GFP/HMVEC versus Mock- $\mathrm{N}^{\mathrm{IC}}$-GFP/HMVEC were tested. IL-6 and IL-8 in Mock and DN-MAML1 are normalized to those in $\mathrm{N}^{\mathrm{IC}}-\mathrm{GFP} / \mathrm{HMVEC}$. Lower. confirmed expression of DN-MAML-1 in DN-MAML-1-N $\mathrm{IC}_{-}$GFP/HMVEC. (C) Increased expression of ICAM-1 in $\mathrm{N}^{\mathrm{IC}}$-GFP/HMVEC versus GFP/HMVEC and $\mathrm{N}^{\mathrm{IC}}$-GFP/HAEC versus GFP/HAEC was tested by immunoblotting assay. $\beta$-actin was used as loading control. Quantitative data was based on results of three independently performed experiments. (D) Detection of production of IL-6, IL-8, RANTES and IL-1a by $\mathrm{N}^{\mathrm{IC}}-\mathrm{GFP} /$ HAEC versus GFP/HAEC by Human Multi-Analyte Elisarray Kit. Supernatants from $\mathrm{N}^{\mathrm{IC}}$ GFP/HAEC versus GFP/HAEC were normalized by cell number. Data are presented as mean \pm SD of three independently performed experiments. IL-1a was undetectable. 


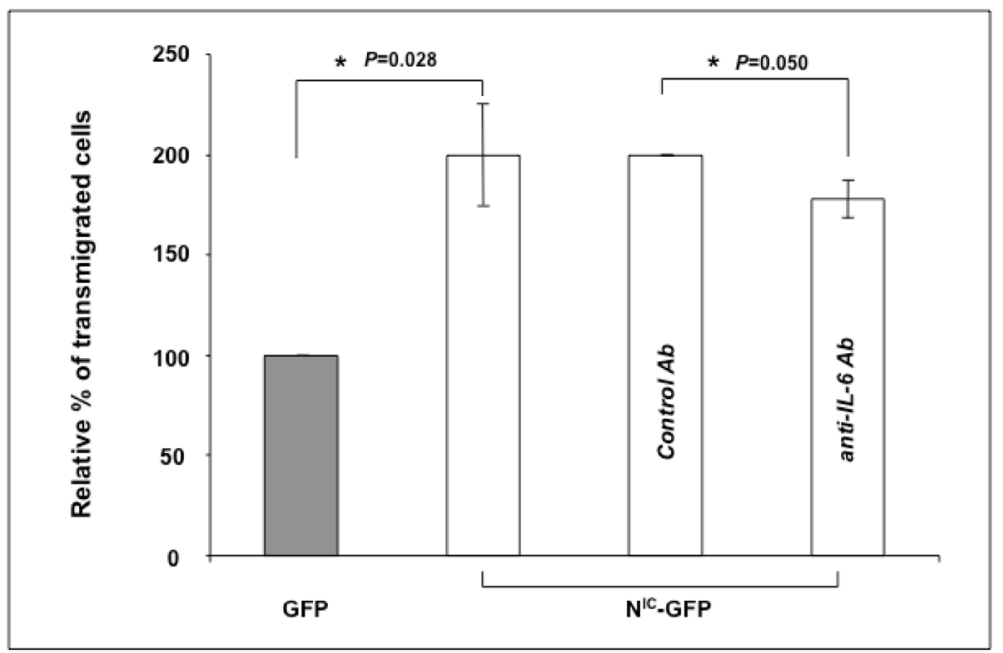

Figure 5.

Activation of Notch1-induces monocyte transendothelial migration in vitro, at least in part, via IL-6. Activation of Notch1 pathway enhanced monocyte transendothelial migration. More THP-1 cells passed through $\mathrm{N}^{\mathrm{IC}}$-GFP/HMVEC versus GFP/HMVEC monolayers in transwell. Blockade of IL-6 significantly inhibited active Notch1-induced monocyte transendothelial migration, though not to baseline level. Numbers of THP-1 cells transmigrated GFP/HMVEC monolayers were set as "100\%" and those passed through $\mathrm{N}^{\mathrm{IC}}$. GFP/HMVEC monolayers were compared. Transmigrated THP-1 cells in isotype-matched control Ab and anti-IL-6 blocking Ab treated wells were normalized with those in non-Ab wells. Data are presented as mean \pm SD of three independently performed experiments. 